\title{
Synthesis of Tetracyclic 5-Azaindole Analogues by Palladium-Catalyzed Sequential Annulation
}

\author{
Nack-Do Sung, ${ }^{\dagger}$ Ok-Kyung Yang, Song Su Kang, and Eul Kgun Yum ${ }^{*}$ \\ ${ }^{\dagger}$ Division of Applied Biology and Chemistry, Chungham National Liversity, Yusung. Daejeon 305-76t. Korea \\ Department of Chemistry, Chmgnam National Lniversity; Yasung, Daejeon 305-764. Korea \\ Received Mon,+ 2004
}

\begin{abstract}
Tetracyclic 5-azaindole analogues were prepared by palladium-catalyzed sequential annulation of benzylidene(3-iodopyridinyl-4-yl)amine and I-aryl substituted internal alkynes under $\mathrm{Pd}(\mathrm{OAc})_{2}, n-\mathrm{Bu}_{4} \mathrm{NCl}$, and $\mathrm{Et}_{3} \mathrm{~N}$ at $120^{\circ} \mathrm{C}$. The synthetic procedure showed possible diversification of tetracyclic 5 -azaindole analogues by varying the $\mathrm{l}$-aryl substituent in internal alkynes.
\end{abstract}

Key Words : Tetracyclic 5-azaindole analogues, Palladium, Catalyst, Annulation, 1-Aryl alkynes

\section{Introduction}

Heteroannulated indole and quinoline alkaloids constitute an important class of natural compounds due to their biological activities, which are based mostly on their affinity toward DNA. ${ }^{1.2}$ Therefore, these compounds play crucial roles as potential leads for the discovery of biologically active substances..$^{3}$ Although isosteric replacements of tetracyclic indole derivatives have strong antibacterial, antimycotic, and antitumor activities, ${ }^{4}$ few synthetic methods for tetracyclic azaindole analogues have been reported in the literature. ${ }^{5.0}$ Recently, our group and Larock's group have reported effective annulation methods ${ }^{?}$ using internal alkynes to prepare a variety of condensed heterocycles, such as indoles, ${ }^{8}$ isoindolo[2.1-a]indoles, ${ }^{9}$ carbolines, ${ }^{10}$ azaindoles, ${ }^{11}$ pyrroloquinolines, ${ }^{12}$ and pyridopyrrolo[2.1-a] isoindoles. ${ }^{1.7}$ Our continued interest is to find prospective bioactive heteroannulated indole analogues. Chemical isosteric replacements of biologically active substances often have improved biological properties, such as potency, selectivity, toxicity, and metabolic stability. ${ }^{14}$ In this paper, we describe the facile preparation of tetracyclic 5-azaindole analogues using palladium-catalyzed annulation with internal alkynes.

\section{Results and Discussion}

We examined the palladium-catalyzed reaction of benzylidene(3-iodopyridin-4-yl)amine with various l-aromatic substituted internal alkynes under $5 \mathrm{~mol} \% \mathrm{Pd}(\mathrm{OAc})_{2}, \mathrm{l}$ eq $n-\mathrm{Bu} \mathrm{H}_{4} \mathrm{NCl}, 2 \mathrm{eq}(i-\mathrm{Pr})_{2} \mathrm{NEt}$, and DMr, at $120^{\circ} \mathrm{C}$. The reactions of benzylidene(3-iodopyridin-4-yl)amine with various functionalized internal alkynes provided reasonable yields of tetracyclic 5-azaindole analogues. The results are summarized in lable 1.

The effect of substituents on the aryl group was first examined with several different aryl internal alkynes (entries 1-4). The reaction using the 3 -flworophenyl internal alkyne provided a somewhat higher yield of the desired product as compared to the reaction using 3-methoxyphenyl internal alkyne. However, the total reaction time was quite dependent
Table 1. Synthesis of tetracyclic 5-araindole analogues by palladium-catalyzed annulation

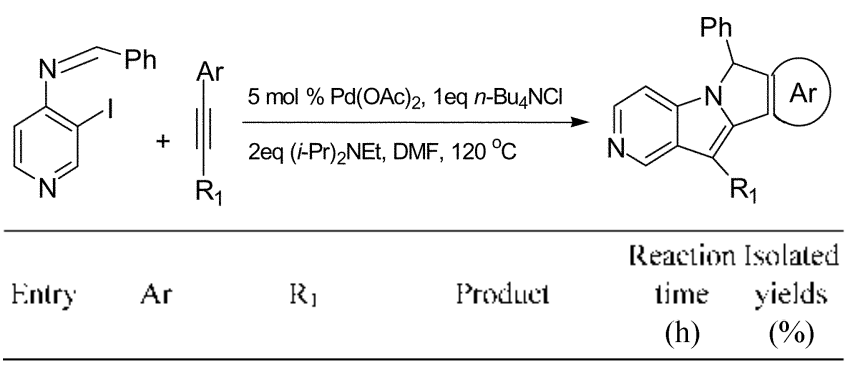

3-FPh




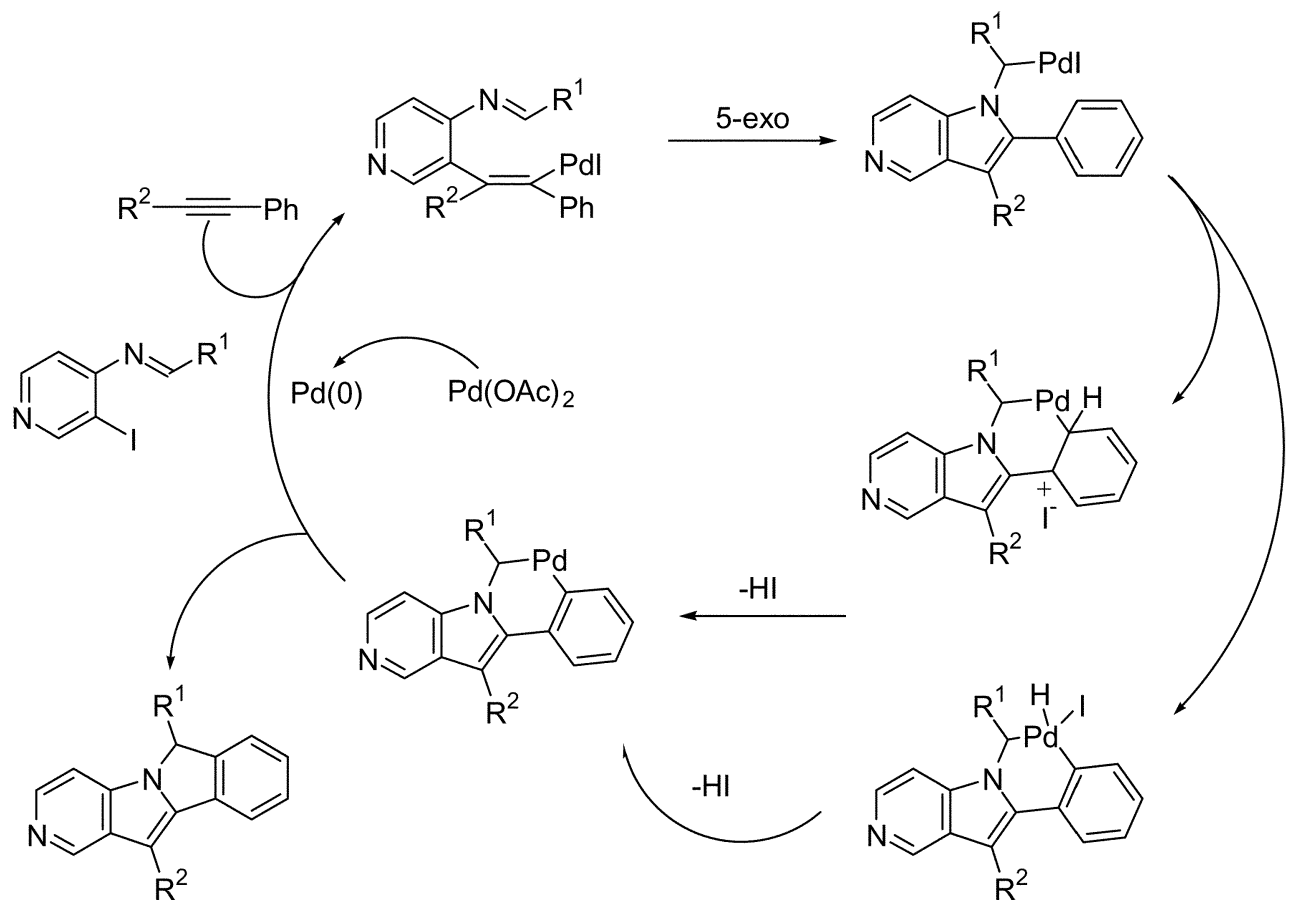

Scheme 1

on the substituent in the phenyl group. Although single tetracyclic 5-azaindole analogues were obtained from the reactions using meta-substituted phenyl internal alkynes, the regioselectivity of the second annulation was quite dependent on the phenyl substituent. Conversely, the reaction using 1 (2-thiophenyl)-1-hexyne provided $20 \%$ of the tetracyclic product owing to deactivation of the palladium catalyst. Finally, the reactions using various alkyl-substituted 1-aryl internal alkynes afforded moderate yields of tetracyclic 5azaindole analogues (entries 5-8). Considering previous annulation results, ${ }^{8.12}$ the annulation might proceed via the route illustrated in Scheme 1. The actual catalyst $P d(0)$ could be formed from $\mathrm{Pd}(\mathrm{OAc})_{2}$ by the reaction medium. $\mathrm{Pd}(0)$ reacts with aryl halides via oxidative addition. The arylpalladium complex coordinates and adds the triple bond of an internal alkyne.

The resulting vinyl palladium intermediate adds the carbon-nitrogen double bonds sequentially. The tetracyclic 5 -azaindole analogues are formed by either electrophilic palladation of the resulting $\pi$-palladium intermediate onto the adjacent aromatic ring or oxidative addition of the neighboring aryl carbon-hydrogen bond of the aromatic ring to the $\pi$-palladium intermediate to form a $\mathbf{P d}$ intermediate, with sequential elimination of $\mathrm{HI}$ by the base, and regeneration of the $\mathrm{l}^{2} \mathrm{~d}(0)$ catalyst by reductive elimination.

\section{Conclusion}

The palladium-catalyzed annulation of benzylidene(3jodopyridin-4-yl)amine with aryl substituted internal alkynes provided tetracyclic 5-azaindole analogues in moderate yields. The annulation reaction showed possible diversification of tetracyclic 5-azaindole analogues with various aryl imines and internal alkynes.

\section{Experimental Section}

IR spectra were obtained using a JASCO FT-JR 410 spectrometer. All the ${ }^{\mathrm{H}} \mathrm{H}$ - and ${ }^{15} \mathrm{C}$-NMR spectra were recorded on a JNM-Al. 400-MHz spectrometer at Chungnam National University. Chemical shitts are given as values relative to tetramethylsilane (TMS) as an internal standard. The GC/MS spectra were obtained on a Shimadzu QP 1000. Melting points were determined on a Mut-TEM apparatus and are uncorrected. Microanalyses were performed at Chungnam National University with a CE Instrument FA 1110. The products were purified by flash chromatography on 230-400-Imesh ASTM 60 silica gel. All the bases, $n$ $\mathrm{Bu} \mathrm{u}_{1} \mathrm{NCl}$, and palladium species were purchased from Aldrich Chemical Co. The other chemicals were used as obtained from commercial sources, unless otherwise noted.

General procedure for the synthesis of the aryl alkynes $^{\text {th }}$. Jo a solution of an iodo- or bromoarene (10.0 mmol) and a terminal alkyne $(12.0 \mathrm{mmol})$ in $\mathrm{Et}_{3} \mathrm{~N}(40 \mathrm{~mL})$ was added $\mathrm{PdCl}_{2}\left(\mathrm{P}^{\mathrm{P}} \mathrm{h}_{3}\right)_{2}(140 \mathrm{mg}, 2 \mathrm{~mol} \%)$ in $\mathrm{Et}_{3} \mathrm{~N}(40$ $\mathrm{mL}$ ). The mixture was then stirred for $5 \mathrm{~min}$, and $\mathrm{Cul}(20$ $\mathrm{mg} .1 \mathrm{~mol} \%$ ) was added. The resulting mixture was heated under a nitrogen atmosphere at $50^{\circ} \mathrm{C}$. 'The reaction mixture was allowed to cool to $\mathrm{rt}$, and the ammonium salt was removed by filtration. The solvent was removed under reduced pressure, and then the residue was removed under reduced pressure. The residue was purified by column chromatography on silica gel to afford the pure alkynes.

Preparation of benzylidene(3-iodo-pyridin-4-yl)amine. A mixture of 4-amino-3-iodopyridine ${ }^{15}$ (1.2 g. $5.5 \mathrm{mmol}$ ). benzaldehyde $(0.58 \mathrm{~g}, 5.5 \mathrm{mmol})$, and a molecular sieve (1 
g) in benzene $(40 \mathrm{~mL}$ ) was refluxed for $24 \mathrm{~h}$ using the DeanStark apparatus to remove the water produced. The reaction mixture was monitored by TLC to establish completion. The reaction mixture was then cooled to rt. and the solvent was removed under reduced pressure. The oily residue was dissolved in a minimal anount of ethanol and cooled. The resulting solid was collected to afford $1.23 \mathrm{~g}(73 \%)$ of the imine as a yellow solid. $\mathrm{mp} 96-97^{\circ} \mathrm{C}$ : IR (KBr) $1644 \mathrm{~cm}^{-1}$. ${ }^{1} \mathrm{H}-\mathrm{NMR}\left(\mathrm{CDCl}_{3}\right) \delta 8.81$ (s lH. - $\mathrm{N}=\mathrm{CH}-$ ), 8.43 (d. $J=4.8$ Hz, IH Ar-H). $8.22(\mathrm{~s}, J=4.8 \mathrm{~Hz}, \mathrm{lH}, \mathrm{ArH}), 7.94-7.92(\mathrm{~m}$. lH, $\mathrm{ArH}$ ), $7.52-7.47(\mathrm{~m}, 4 \mathrm{H} . \mathrm{ArH}) .6 .83(\mathrm{~d} . J=4.8 \mathrm{~Hz} . \mathrm{lH}$. ArH); ${ }^{13} \mathrm{C}-\mathrm{NMR}\left(\mathrm{CDCl}_{3}\right) \delta 162.8$. 159.7, $157.1,149.9$. 134.7, 132.4, 129.4. 129.2. 128.7, 113.8: MS (EI) $(m z) 309$ $\left(\mathrm{M}^{+}+1,1 \mathrm{l}\right) .308\left(\mathrm{M}^{-}, 79\right) .204$ (17). $18 \mathrm{l}(56) .91$ (31). 77 (23), 43 (100): Anal. Calcd for $\mathrm{C}_{13} \mathrm{H}_{3} \mathrm{~N}_{3} \mathrm{I}: \mathrm{C} .46 .78 \mathrm{H}, 2.94$. N. 9.09 . Found: C, 46.81: H. $2.90 ;$ N, 9.05 .

General procedure for the synthesis of tetracyclic 5azaindole derivatives by palladium-catalyzed annulation. Palladium acetate $(6 \mathrm{mg}, 0.025 \mathrm{mmol}), n-\mathrm{Bu} \mathrm{A}_{4} \mathrm{NCl}(139 \mathrm{mg}$. $0.5 \mathrm{mmol}), \mathrm{Et}_{3} \mathrm{~N}$ ( $\left.10 \mathrm{lmg} .1 .0 \mathrm{mmol}\right)$. benzylidene(3-iodopyridin-4-yl)amine ( $154 \mathrm{mg}, 0.5 \mathrm{mmol}$ ). diphenylacetylene (178 mig. $1.0 \mathrm{mmol})$. and DMF $(10 \mathrm{~mL})$ were added to a pressure tube equipped with a stirring bar. After heating the reaction mixture for $11 \mathrm{~h}$ at $120^{\circ} \mathrm{C}$, the resulting solution was diluted with etlyy acetate and washed with saturated aqueous anmonium chloride. The organic layer was dried over $\mathrm{MgSO}_{4}$, filtered, and concentrated. The residue was purified by column chromatography using hexane-ethyl acetate $(1: 1)$. 6.11-Diphenyl-6H-pyrido[3'.4':4.5]pyrrolo[2,1-c] isoindole (1) $(120 \mathrm{mg}, 67 \%)$ was obtained as a yellow: solid. mp 149-150 ${ }^{\circ} \mathrm{C}$ : IR (KBr) 3025. 1602. $1455 \mathrm{~cm}^{-1}:{ }^{1} \mathrm{H}-$ NMR $\left(\mathrm{CDCl}_{\hat{s}}\right) \delta 9.04$ (s. $\left.\mathrm{lH}, \mathrm{ArH}\right) .8 .13(\mathrm{~d} . J=5.6 \mathrm{~Hz}, \mathrm{lH}$. ArH). $7.83(\mathrm{~d}, J=7.6 \mathrm{~Hz}, 1 \mathrm{H}$. ArH). $7.74(\mathrm{~d} . J=4.8 \mathrm{~Hz}, 2 \mathrm{H}$. ArH). 7.37 (t. $J=7.3 \mathrm{~Hz}, 1 \mathrm{H} . \mathrm{ArH}), 7.29-7.24(\mathrm{~m}, 3 \mathrm{H}, \mathrm{ArH})$. 7.22-7.08 (m. 5H. ArH). 6.75 (d, $J=6.0 \mathrm{~Hz}, 1 \mathrm{H} . \mathrm{ArH}$ ), 5.98 (s. $1 \mathrm{H}, \mathrm{ArCH}) ;{ }^{13} \mathrm{C}-\mathrm{NMR}\left(\mathrm{CDCl}_{3}\right) \delta 147.9,142.2 .140 .9$. $139.6,137.6 .136 .5,133.2,130.4 .128 .9,128.8,129.0$. $128.9,128.5,128.2,128.2,126.8,123.8,121.0,109.0$. 104.9. 63.9: MS (EI) $(m z) 358\left(\mathrm{M}^{+} .100\right), 281$ (55), 64 (17): Anal. Calcd for $\mathrm{C}_{76} \mathrm{H}_{18} \mathrm{~N}_{2}: \mathrm{C}, 87.12 ; \mathrm{H} .5 .06 ; \mathrm{N}, 7.82$. Found: C. 87.09 ; H. 5.07: N. 7.83 .

The following compounds were obtained using the above general procedure.

11-n-Butyl-9-fluoro-6-phenyl-6 $H$-pyrido $\left[3^{\prime}, 4^{\prime}: 4,5\right]$ pyrrolo[2,1-a] isoindole (2). The compound (2) was obtained as brown oil in $45 \%$ yield with a $24 \mathrm{~h}$ reaction (Table l. entry 2 ). IR $(\mathrm{KBr}) 3029.1604,1467 \mathrm{~cm}^{-1}:{ }^{1} \mathrm{H} \mathrm{NMR}\left(\mathrm{CDCl}_{3}\right) \delta 8.81$ (s. $1 \mathrm{H}, \mathrm{ArH}$ ). 8.00 (s. $\mathrm{lH}, \mathrm{ArH}$ ). 7.48 (d. $J=7.2 \mathrm{~Hz}, \mathrm{lH}$. ArH). 7.36-7.20 (m. 4H, ArH), 7.07-6.09 (n, 2H. ArH). 6.86-6.81 (m, 2H. ArH). 5.99 (s, IH. ArCH), 2.98 (d. $J=7.6$ $\left.\mathrm{Hz}, 2 \mathrm{H} . \mathrm{CH}_{2}\right) .1 .71\left(\mathrm{q}, 2 \mathrm{H}, \mathrm{CH}_{2}\right), 1.39\left(\mathrm{~m}, 2 \mathrm{H}, \mathrm{CH}_{2}\right), 0.89$ (t. $\left.J=7.2 \mathrm{~Hz}, 3 \mathrm{H} . \mathrm{CH}_{3}\right) ;{ }^{13} \mathrm{C} \mathrm{NMR}\left(\mathrm{CDCl}_{3}\right) \delta 162.9,142.6$. $1+2.1,1+0.2 .137 .8,136.6,132.9 .131 .0,128.9,128.6$. $126.9,125.2,117.1,114.5,114.2,108.3,108.1 .63 .7,33.2$. $24.1,22.6,14.0$; MS (EI) $(m z) 356\left(\mathrm{M}^{-}, 6\right) .313(22) .149$ (13), 70 (18). 43 (100): Anal. Calcd for $\mathrm{C}_{2} \mathrm{H}_{2} \mathrm{~N} \mathrm{~N}_{2} \mathrm{~F}$ : C. 80.87: H, 5.94; N. 7.86. Found: C, 80.85: H, 5.96; N. 7.86
11-n-Butyl-7-methoxy-6-phenyl-6H-pyrido $\left[3^{\prime}, 4^{\prime}: 4,5\right]-$ pyrrolo[2,1-a] isoindole (3). The compound (3) was obtained as yellow solid in $34 \%$ yield with a $12 \mathrm{~h}$ reaction (Table 1, entry 3). $\mathrm{mp}=150-151^{\circ} \mathrm{C}: \mathrm{IR}(\mathrm{KBr}) 2928.1466$ $\mathrm{cm}^{-1}$ : ${ }^{1} \mathrm{H} \mathrm{NMR}\left(\mathrm{CDCl}_{3}\right) \delta 8.90(\mathrm{~s}, 1 \mathrm{H}, \mathrm{ArH}), 8.10(\mathrm{~d}, J=5.6$ Hz. IH. ArH), 7.33-7.30 (m. 3H. ArH), 7.11-7.09 (m, 2H, $\mathrm{ArH}), 6.79-6.74$ (m. 2H. ArH ). 6.04 (s. IH. $\mathrm{ArC} \underline{\mathrm{H}}$ ). 3.87 (s. $\left.3 \mathrm{H} . \mathrm{OCH}_{3}\right), 3.09$ (t. $\left.J=7.6 \mathrm{~Hz}, 2 \mathrm{H} . \mathrm{CH}_{2}\right), 1.82(\mathrm{q}, J=7.6$ Hz. $\left.2 \mathrm{H}, \mathrm{CH}_{2}\right) .1 .49\left(\mathrm{~m}, 2 \mathrm{H} . \mathrm{CH}_{2}\right) .0 .99$ (t. $J=7.2 \mathrm{~Hz} .3 \mathrm{H}$, $\left.\mathrm{CH}_{3}\right):{ }^{13} \mathrm{C}$ NMR $\left(\mathrm{CDCl}_{3}\right) \delta 160.1,142.9 .140 .6 .139 .8$, 139.2, 138.6. 136.6. 132.6. 129.7, 129.1. 128.5, 126.9. $124.7,113.1$. 108.1, 106.8. 104.8. 63.7, 55.5, 33.3, 24.1, 22.6. 14.0: MS (EI) $(m z) 368\left(\mathrm{M}^{+} .27\right), 325(100) .293(26)$, 281 (17). 205 (13), 140 (11); Anal. Calc. for $\mathrm{C}_{35} \mathrm{H}_{24} \mathrm{~N}_{2} \mathrm{O}$ : C, 81.49: H. 6.57: N, 7.60. Found: C. 81.46; H, 6.59: N, 7.61

Compound 4 . The compound (4) was obtained as brown oil in $20 \%$ yield with with a $24 \mathrm{~h}$ reaction (Table l. entry 4 ). IR (KBr) 3041. 2925, 1552. $1454 \mathrm{~cm}^{-1},{ }^{1} \mathrm{H} \mathrm{NMR}\left(\mathrm{CDCl}_{3}\right) \delta$ 8.84 (s. $1 \mathrm{H}, \mathrm{ArH}$ ), 8.07 (d. $J=5.6 \mathrm{~Hz}, \mathrm{lH} . \mathrm{ArH}$ ). $7.35-7.32$ (m. $4 \mathrm{H}, \mathrm{ArH}), 7.18-7.14$ (m. $2 \mathrm{H}, \mathrm{ArH}), 6.88(\mathrm{~d}, J=4.8 \mathrm{~Hz}$. lH. $\mathrm{ArH}$ ). 6.75 (d. $J=5.6 \mathrm{~Hz}, \mathrm{lH}, \mathrm{ArH}), 6.10$ (s. $1 \mathrm{H}$, $\left.\mathrm{ArCH}_{2}\right), 2.95$ (t. $\left.J=7.6 \mathrm{~Hz}, 2 \mathrm{H}, \mathrm{CH}_{2}\right), 1.84$ (q. $J=7.6 \mathrm{~Hz}$, $\left.2 \mathrm{H} . \mathrm{CH}_{2}\right) .1 .46\left(\mathrm{~m}, 2 \mathrm{H} . \mathrm{CH}_{2}\right), 0.99\left(\mathrm{t} . J=7.2 \mathrm{~Hz}, 3 \mathrm{H}_{2} \mathrm{CH}_{3}\right)$ : ${ }^{13} \mathrm{C}$ NMR $\left(\mathrm{CDCl}_{3}\right) \delta 141.7 .139 .8 .137 .7 .137 .2,129.6$, $129.1,128.6 .128 .4 .126 .8 .126 .7,125.8 .121 .3,107.3$, $105.5,104.9,63.3,32.6 .24 .6,22.7,14.1 ; \mathrm{MS}$ (EI) (mz) 344 $\left(\mathrm{M}^{-}, 17\right) .30 \mathrm{l}(40), \mathrm{l} 49$ (10). 105 (15), 43 (100); Anal. Calcd for $\mathrm{C}_{2} \mathrm{H}_{2, \mathrm{~N}} \mathrm{~N} \mathrm{~S}: \mathrm{C}, 76.71 ; \mathrm{H}, 5.85$ : N. 8.13. Found: C, 76.69; H. $5.83 ;$ N. 8.15.

11-Methyl-6-phenyl-6H-pyrido[3',4':4,5]pyrrolo[2,1-a]isoindole (5). The compound (5) was obtained as a yellow solid in $55 \%$ yield with a $10 \mathrm{~h}$ reaction (Table 1 , entry 5 ). $\mathrm{mp}$ $162.163{ }^{\circ} \mathrm{C}$ : IR $(\mathrm{KBr}) 2916,1449 \mathrm{~cm}^{-1}$. ${ }^{1} \mathrm{H}-\mathrm{NMR}(\mathrm{CDCl}) \delta$ $8.26(\mathrm{~s}, 1 \mathrm{H} . \mathrm{ArH}) .8 .18(\mathrm{~d} . J=5.6 \mathrm{~Hz}, 1 \mathrm{H} . \mathrm{ArH}), 7.84($ d. $J=$ 7.6 Hz. $1 \mathrm{H}, \mathrm{ArH}$ ), 7.48-7.43 (m. $2 \mathrm{H}, \mathrm{ArH}) .7 .33-7.26(\mathrm{~m}$, 5H. ArH). 7.14-7.12 (m. 2H. ArH). 6.15 (s. 1H, ArCH), 2.58 (s, 3H. $\left.\mathrm{CH}_{3}\right):{ }^{13} \mathrm{C}-\mathrm{NMR}\left(\mathrm{CDCl}_{3}\right) \delta 147.3,143.0 .138 .4$, 138.3, 138.2. 132.6. 131.4. 130.1, 129.2. 128.7, 128.5, $128.0,127.0 .124 .1 .121 .5 .113 .9 .101 .7,64.6 .8 .8$; MS (EI) (mz) $297\left(\mathrm{M}^{-}+\mathrm{l}, 12\right), 296\left(\mathrm{M}^{+} .100\right) .219(39) .146(14)$; Anal. Calcd for $\mathrm{C}_{21} \mathrm{H}_{16} \mathrm{~N}_{2}:$ C. 85.11 : H. 5.44; N, 9.45. Found: C. 85.07: H. 5.46; N. 9.47 .

11-Hydroethyl-6-phenyl-6H-pyrido[3', $\left.\mathbf{3}^{\prime}: 4,5\right]$ pyrolo[2, 1-a] isoindole (6). The compound (6) was obtained as a yellow solid in $43 \%$ yield with a $20 \mathrm{~h}$ reaction (Table $\mathrm{l}$ entry 6). $\mathrm{mp}=145.148{ }^{\circ} \mathrm{C}$ : IR $(\mathrm{KBr}) 3397,2924.2854 \mathrm{~cm}^{-1} .{ }^{1} \mathrm{H}$ NMR (DMSO) $\delta 8.95$ (s. lH. ArH). 8.09 (d, $J=5.2 \mathrm{~Hz}, 1 \mathrm{H}$, $\operatorname{ArH}) .7 .95($ d. $J=7.2 \mathrm{~Hz}, 1 \mathrm{H}$. ArH). $7.56(\mathrm{~d}, J=5.2 \mathrm{~Hz} .2 \mathrm{H}$, ArH), 7.29-7.27 (m, 4H. ArH), 7.13 (d. $J=5.6 \mathrm{~Hz} .2 \mathrm{H}$, $\mathrm{ArH}), 6.82$ (d. $J=6.0 \mathrm{~Hz}, \mathrm{ArH}$ ). 6.47 (s. $1 \mathrm{H}$. $\left.\mathrm{ArCH}_{2}\right) .4 .97$ (br. 1 H. $-\mathrm{OH}$ ), 3.83 (t. $\left.J=7.2 \mathrm{~Hz}, 2 \mathrm{H} . \mathrm{CH}_{2}\right), 3.27$ (t. $J=6.8$ Hz. $2 \mathrm{H} . \mathrm{CH}_{2} \mathrm{OH}$ ): ${ }^{13} \mathrm{C}$ NMR (DMSO) $\delta 146.8 .142 .5,140.4$, 139.9. 138.5. 135.7. 130.5. 129.0, 128.9. 128.5, 128.4. $128.1,127.7,126.7,124.0 .121 .3,103.9 .63 .1,61.7 .27 .9$; MS (EI) (mz) 326 (M+. 26), 295 (100), $218(8), 147(8)$; Anal. Calcd for $\mathrm{C}_{22} \mathrm{H}_{18} \mathrm{~N}_{2} \mathrm{O}$ : C, 80.96; H. 5.56: N. 8.58 . Found: C, 80.94; H. 5.58: N, 8.58. 
11-Methoxymethyl-6-phenyl-6H-pyrido[3',4':4,5]pyrrolo$[2,1-a]$ isoindole (7). The compound (7) was obtained as a yellow solid in $51 \%$ yield with a 20 h reaction. $\mathrm{mp}=134$ $135^{\circ} \mathrm{C}$; IR (KBr) $2930,1454 \mathrm{~cm}^{-1}$; ${ }^{1} \mathrm{H}$ NMR (DMSO) $\delta$ 8.94 (s, IH. Ar-H). 8.07 (d, $J=6.0 \mathrm{~Hz} . \mathrm{IH}, \mathrm{ArH}), 8.01$ (d, $J$ $\left.=7.6 \mathrm{~Hz} .1 \mathrm{H}, \mathrm{ArH}_{\mathrm{H}}\right) .7 .48$ (t. $\left.\mathrm{lH} . \mathrm{ArH}_{\mathrm{H}}\right) .7 .37 .7 .28(\mathrm{~m}, 5 \mathrm{H}$. $\mathrm{A}_{\mathrm{HH}}$ ). 7.13-7.11 (nt. 2H, ArH), 6.85 (d, $J=6.0 \mathrm{~Hz} .1 \mathrm{H}$. $\mathrm{ArH}) .6 .58$ (s, IH. $\mathrm{ArCH}$ ). 4.97 (s, 2H. CH. $\mathrm{CH}_{2} \mathrm{O}$ ). 3.38 (s, 3H. $\left.\mathrm{OCH}_{3}\right) ;{ }^{13} \mathrm{C}$ NMR (DMSO) $\delta 147.3 .142 .6,141.7,140.4$. $138.2,135.7 .129 .8,128.9,128.7 .128 .6,128.4,128.3$. $126.7,124.1,122.1,105.1,103.0,63.9,63.3 .56 .9$. MS (ED) (mz) $326\left(\mathrm{M}^{+}, 50\right) .295$ (100). 147 (26). 77 (14). 51 (10): Anal. Calcd for $\mathrm{C}_{2} \mathrm{H}_{18} \mathrm{~N}_{2} \mathrm{O} ; \mathrm{C} .80 .96 ; \mathrm{H}, 5.56 ; \mathrm{N} .8 .58$. Found: C. $80.94 ;$ H, $5.57 ;$ N. 8.59 .

6-Phenyl-6H-pyrido[3',4':4,5]pyrrolo[2,1-a]isoindole-11carboxylic acid ethyl ester (8). The compound (8) was obtained as a brown solid in $45 \%$ yield with a $27 \mathrm{~h}$ reaction. $\mathrm{mp}=152.155^{\circ} \mathrm{C}$; IR (KBr) $1684 \mathrm{~cm}^{-1} ;{ }^{1} \mathrm{H}$ NMR (DMSO) $\delta$ 8.68 (d. $J=7.6 \mathrm{~Hz}, \mathrm{IH} . \mathrm{ArH}), 8.60$ (d. $J=7.6 \mathrm{~Hz} .1 \mathrm{H}, \mathrm{ArH})$. $8.19(\mathrm{~s}, \mathrm{lH} . \mathrm{ArH}) .8 .01(\mathrm{~d} . J=5.6 \mathrm{~Hz}, 1 \mathrm{H}, \mathrm{ArH}) .7 .59(\mathrm{t}, J=$ $7.2 \mathrm{~Hz}, \mathrm{IH} . \mathrm{ArH}), 7.51$ (t. $J=7.2 \mathrm{~Hz}, 1 \mathrm{H}, \mathrm{ArH}) .7 .41-7.31$ (m. 3H. ArH). 7.23 (d, $J=7.2 \mathrm{~Hz} .1 \mathrm{H}, \mathrm{ArH}$ ), 7.19-7.15 (m. $2 \mathrm{H}, \mathrm{ArH}$ ). 6.74 (s. IH. $\mathrm{ArCH}_{2}$ ). 4.47 (q. $J=7.2 \mathrm{~Hz} .2 \mathrm{H}$. $\left.\mathrm{OCH}_{2}\right) .1 .47\left(\mathrm{t}_{,} J=7.2 \mathrm{~Hz}, 3 \mathrm{H} . \mathrm{CH}_{3}\right):{ }^{13} \mathrm{C} \mathrm{NMR}$ (DMSO) $\delta$ 164.2, 150.9. 147.8, 141.1, 136.1. 129.8, 129.1, 129.0. 128.7, 128.6. 128.5, 128.2, 127.4. 126.7, 125.6, 125.2 . 123.2, 64.6. 60.1. 49.8, 14.4; MS (EI) $(m z) 355\left(\mathrm{M}^{-}+1,34\right)$. $354\left(\mathrm{M}^{-}, 100\right), 325(58), 309(29), 282(38), 281(46) .140$ (23), $126(20)$.

Acknowledgement. This work was supported by a grant (No. RI1-2002-100-03002-2) from the ERC progran of the Korea Science \& Engineering Foundation.

\section{References}

1. Knölker. H.-J.: Reddy. K. R. Chem. Rev. 2002. 102. 4303.

2. Katritzky. A. R.: Rees. C. W.: Scriven. E. F. V. Conprehensive Heterocycic Chentistry II, Elsevier Science Ltd., 1996; Vol. 2. pp $1-117$

3. Kirsch. G. H. Curren Organic Chentisny 2001. 5, 507.

4. Pierre. A.: Atass. G.: Devissaguet. M.: Bisagni. E. Dnugs Fut 1997. 22. 53 and references therein.

5. Desarbrec. E.: Merour. J.-Y. Heterocycles 1995. H. 1987.

6. Merour. J.-Y.; Joseph. B. Cinrent Organic Chentistry 2001.5.471.

7. Reviews: (a) Larock. R. C. J. Organontet. Chem. 1999. 576, 111 (b) Larock, R. C. Pure and Appl. Chem. 1999, 71. 1435. (c) Li. J. J.: Gribble. G. W. Palladium in Heterocychic Chemistry. Pergamon: 2000): pp 143-146.

8. (a) Larock. R. C.: Yum. E. K. J. Am. Chem. Soc. 1991. 113. 6689 (b) Larock, R. C. Yum. E. K.; Rettik. D. J. Org. Chent. 1998. 63. 7652. (c) Hong, K. B.: Lee, C. W.: Yum, E. K. Tetrahedron Lett. 2004. $+5,693$

9. (a) Roesch. K. R.: Larock. R. C. Org. Lett. 1999. 1. 1551. (b) Roesch. K. R.: Larock. R. C. J. Org. Chem. 2001. 66. 412.

10. (a) Zhang. H.: Larock. R. C. Org. Lett 2002. 4. 3035. (b) Zhang. H. Larock, R. C. J. Ong. Chem. 2003, 68. 5132.

11. (a) Park, S. S.; Choi. J.-K.: Yum, E. K; Ha. D.-C. Tetrohedron Lett. 1998, 39, 627. (b) Chi. S. M.: Choi, J.K.: Yum. E. K. Chi. D. Y. Tetrahedron Lett. 2000. 11.919. (c) Lee. M. S.: Yum. E. K. Bull. Korean Chem. Soc. 2002. 23.535.

12. (a) Kang. S. K.: Park. S. S.: Kim. S. S.: Choi. T.-K.: Yum. E. K. Tetrahedhon Lett. 1999, 40. 4379. (b) Yum. E. K; Kang. S. S: Kim, S. S.: Choi. J.-K, Cheon, H. G. Bioong. Med. Chem. Lett. 1999. 9, 1819. (c) Gee, M. B.; Lee, W. J.: Yum. E. K. Bull. Korean Chem. Soc. 2003. 24. 1193 , (d) Lee. W. J.: Gee. M. B.: Yum. E. K. Heterocycles 2003. 60. 1821 .

13. Kang. S. S.: Yum. E. K.: Sung. N.-D. Heterocycles 2003. 60. 2727 .

14. Williams. D. A.: Lemke. T. L. Foye's Principles of Medicinal Chemistry, $5^{\text {dh }}$ ed Lippincott Williams \& Wilkins: 2002.

15. (a) Marsais. F.: Gogard. A.: Queguiner. G.J. Heterocycl. Chem 1989. 26. 1589. (b) Queguiner. G. J. Heterocycl. Chem. 2000. 37.615. 